\title{
EVALUACIÓN INTEGRAL DE SEIS VARIEDADES DE ACEITUNA (Olea europaea $L$ ) ACEITERAS DEL BANCO DE GERMOPLASMA DE OLIVO DEL INPREX - UNJBG - TACNA
}

\author{
COMPREHENSIVE EVALAUTION OF SIX OIL-PRODUCING OLIVE VARIETIES \\ (Olea europaea L.) SUPPLIED BY THE GERMOPLASM BANK OF IMPREX- \\ UNJBG, TACNA
}

\author{
Enrique A. De Florio Ramirez'; Liliana Lanchipa Bergamini ${ }^{2}$
}

\begin{abstract}
RESUMEN
En el presente trabajo se determina la aptitud de seis variaedades de aceituna para la obtención de aceite de oliva(olea europaea.1), correspondiente al banco de germoplasma del IMPREX - UNJBG. Se analizó las sigueintes caracteristicas del fruto: Índice de madurez, la relación Pulpa-hueso, entre medio y alta. El porcentaje de aceite en base a la húmedad y por variedad fue: Sevillana (25.6\%), Picual (19.72\%), Pendolino (20.10\%), Azaradj (21.73\%), Manzanilla (28.78\%), Campanil (26.61\%). Al aceite de oliva extra virgen, obtenido de las seis variedades se realizó el análisis físico químicos: Índice acidez, índice de Peróxidos, $K_{270}$ y el análisis sensorial, encontrándose todos dentro de la norma que los sindica como tal. Por $1 a$ cantidad y la calidad física, química de aceite producido se ha considerado las de mejor aptitud aceitera la Sevillana y Pendolino.
\end{abstract}

Palabras clave: aceituna, aceite de oliva, Germoplasma

\section{ABSTRACT}

\begin{abstract}
The estudy detrmines the feasibility of the obtention of olive oil from six olive varieties (olea europaea L) supplied by the Germoplasm Bank of INPREX-UNJBG, Tacna, Perú. Important fruit characteristics of these varieties were analyzed such as Maturity index (In the optimum range of 2.3-3.5). Pulp-Pit Ratio (Middle-high range). Oil percentage, wet-basic: Sevillana (25.6\%), Picual (19.72), Pendolino (20.10\%), Azaradj (21.73\%), Manzanilla (28.18\%), Campanil (26.61\%). To the extra-virgin oil obtained from these six varieties were made the following test: Physical -chemical analisys, acid index, Peroxide index, K270, and Sensorial evaluation. The results obtained conforms to the form for these type of oil. The best varieties upon the basis of the the amount, physical and chemical quality of the oil obtains, were. Sevillana, (good for oil and non-oil purposes) and Pendolino
\end{abstract}

Keywords: Olive, Olive oil, Germoplasm Bank

\section{I.- INTRODUCCIÓN}

El olivo es la especie frutícola más importante del departamento de Tacna, considerado este como el primer productor. En el año 2003 se registró una producción de 23462 TM que equivalen al $61,7 \%$ de la población Nacional y la perspectiva de incrementarse es mayor, en tanto que se ha sembrado, el año 2002 y 2003 aproximadamente 1000 has, además, cuenta con la productividad más alta del país, con 6.4 TM/Ha (Ministerio de Agricultura, 2008). Se tiene también que el $15 \%$ de la producción se destina para la industria del aceite de oliva y el $85 \%$ para procesamiento en aceituna de botija (Díaz, 1988).
Contraria a la tendencia mundial donde es mayor la producción de Aceite.

El INPREX (Instituto de Proyección y Extensión Social de la UNJBG) posee en el programa de fruticultura, un banco de germoplasma que contiene 31 variedades de olivo edafoclimáticas, adaptadas en un área de 0.30 Has.

Una variedad edafoclimáticas adaptada a su medio es, menos susceptible a los factores biológicos y abióticos, lo que tiene una repercusión muy directa en la calidad del aceite de oliva (COI 2002, según menciona Alferez (2007)). Por otro lado, es necesario la caracterización (agronómica, 
fenológica, pomológica y relativa a la calidad del aceite) de las variedades para conocer mejor los recursos genéticos, con vistas a utilizarlas correctamente, permitiendo así, obtener productos oleicos de calidad (COI 2002, Alférez (2007)), del mismo modo, se hace necesario también una caracterización física, química de las variedades de olivo del banco de germoplasma, programa de fruticultura INPREX, para determinar la idoneidad como materia prima en los diferentes procesos de post cosecha ya que la adaptación edafoclimática podría generar un cambio de las características de la zona de donde es originaria.

\section{OBJETIVO}

Determinar la caracterización tecnológica de las variedades de germoplasma del INPREX y evaluar las seis variedades de aceituna (olea europeae l) aceiteras del banco de germoplasma de olivo del INPREX-UNJBG Tacna.

\section{II.- MATERIALES Y MÉTODOS}

La presente investigación se realizó en los laboratorios de FAIA - UNJBG.

\subsection{Materia Prima}

Las muestras de aceitunas se obtuvieron del Banco de Germoplasma del IMPREX.: Picual, Sevillana, Azaredj, Manzanilla, Pendolita, campanil.

\subsection{Materiales y Métodos}

\subsubsection{Materiales}

- Material de vidrio: Vaso de precipitados, Erlenmeyer, Pipetas, Pera de Separación Etc.

- Reactivos: Ioduro de Potasio, Tíosulfato Sódico, Ácido acético, Solución de almidón, Alcohol etílico, Éter Etílico, Fenolftaleína, Etc.

- Equipos: Sistema Soxhlet. Licuadora, Baño María, Centrifuga. Espectrofotómetro UV.

\subsubsection{Métodos}

\section{- Análisis Materias Primas}

Índice de madurez (Frias 1991)

Peso promedio (Frias 1991)

Relación pulpa/hueso. (Frias 1991)

Contenido de Humedad. (Frias 1991)

Contenido de Aceite (Frias 1991)
Índice de Peróxidos.(Frias 1991)

Coeficiente de extinción (Frias 1991)

Acidez Libre. (Frias 1991)

- Análisis sensorial del aceite de oliva virgen (Frias, 1991).

\subsection{Diseño experimental}

El diseño experimental del presente trabajo se muestra en el Gráfico $\mathrm{N}^{\circ} 01$ y Gráfico $\mathrm{N}^{\circ} \mathrm{O} 2$, se muestra el diagrama de operaciones para la obtención del aceite de oliva virgen.

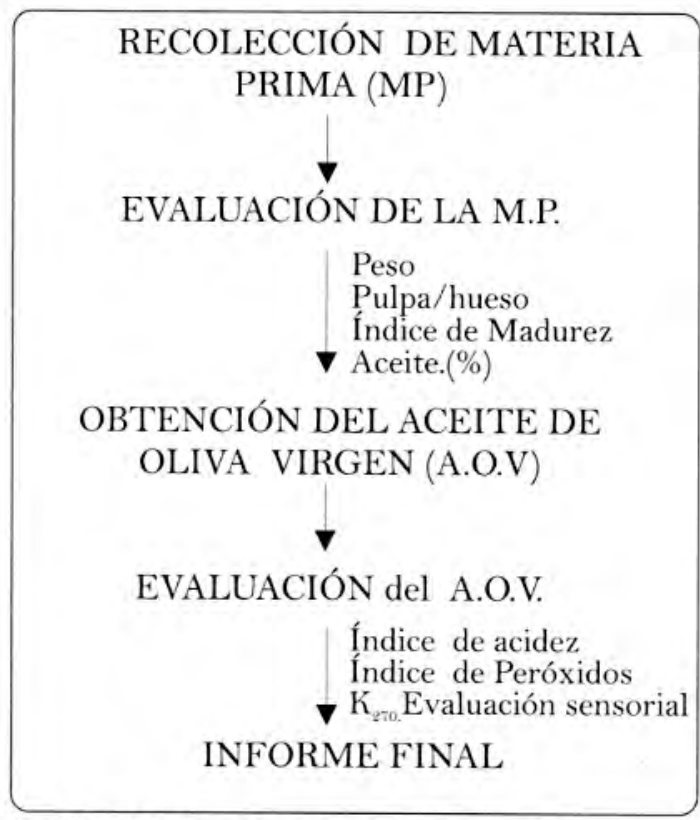

Gráfico $\mathbf{N}^{\circ} \mathbf{0 1}$. Diagrama experimental

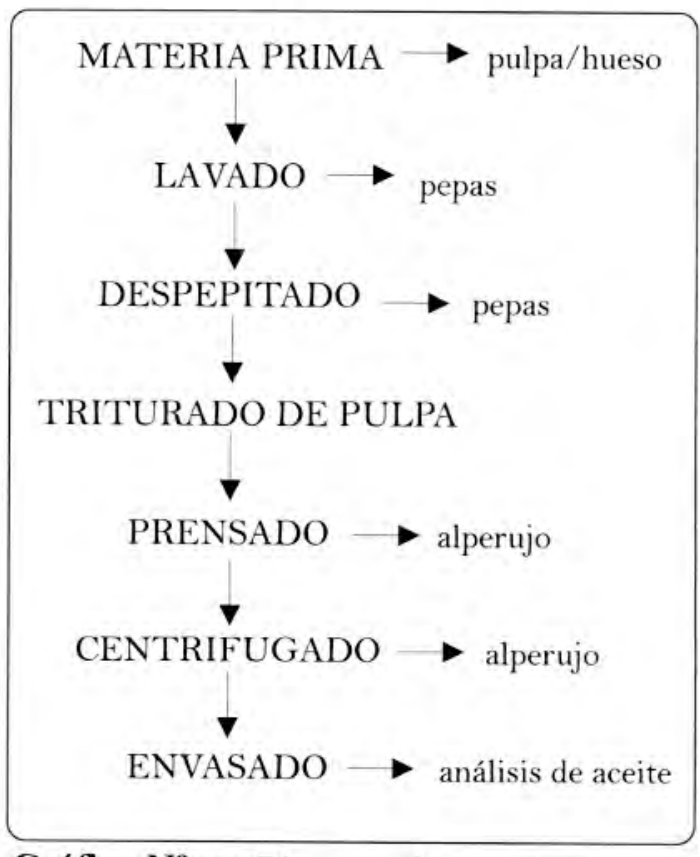

Gráfico $\mathbf{N}^{\mathbf{o}} \mathbf{0 2}$. Diagrama de operaciones para la obtención del aceite de oliva virgen. 


\section{III.- RESULTADOS Y DISCUSIÓN}

\section{A.-ACEITUNAS}

\section{1.-Características del fruto}

La Tabla $\mathrm{N}^{\circ} 01$ indica que las mayorías de las muestras están envero considerada en el momento fisiológico de máxima producción de aceite. Posteriormente varía por otras razones. En relación a la pulpa - hueso las variedades Azeradj y Pendolino son más bajos y se pueden considerar como idóneas para la elaboración de aceite.

Tabla $\mathbf{N}^{\circ} \mathbf{0 1}$. Características del fruto

\begin{tabular}{|l|r|r|r|}
\hline Variedad & $\begin{array}{c}\text { índice } \\
\text { madurez }\end{array}$ & Pulpa/hueso & $\begin{array}{c}\text { Humedad } \\
(\%)\end{array}$ \\
\hline Picual & 3,6 & 8,5 & 71,7 \\
\hline Azeradj & 4,2 & 5,2 & 63,7 \\
\hline Sevillana & 3 & 9,6 & 61,4 \\
\hline Manzanilla & 3,5 & 8,7 & 69,4 \\
\hline Pendolino & 2,87 & 6 & 51,3 \\
\hline Campanil & 2,74 & 8,5 & 71,7 \\
\hline
\end{tabular}

\section{2.- Contenidos del Aceite}

En la Tabla $\mathrm{N}^{\circ} \mathrm{O} 2$ el valor de las grasas no son buenas siendo las mejores la sevillana y Pendolino.

Tabla $\mathbf{N}^{\mathbf{0}} \mathbf{0 2}$. Contenido de aceite en las variedades de aceitunas estudiadas

\begin{tabular}{|l|c|c|}
\hline \multirow{2}{*}{ Variedad } & \multicolumn{2}{|c|}{ Aceite (\%) } \\
\cline { 2 - 3 } & BS & BH \\
\hline Sevillana & 42,01 & 25,76 \\
\hline Picual & 27,49 & 19,72 \\
\hline Pendolino & 29,39 & 20,10 \\
\hline Azarajad & 34,63 & 21,73 \\
\hline Manzanilla & 41,45 & 28,78 \\
\hline Campanil & 37,18 & 26,61 \\
\hline
\end{tabular}

\section{B.-ACEITE}

\section{1.-Acidez}

Como muestra el la Tabla $\mathrm{N}^{\circ}$ 03, los aceites tienen un valor que lo determina como extra virgen. Los más bajos corresponde a las variedades de Manzanilla y Sevillana.

Tabla $\mathbf{N}^{\mathbf{0}} \mathbf{0 3}$. Porcentaje de acidez de las muestras

\begin{tabular}{|l|c|}
\hline \multicolumn{1}{|c|}{ Variedad } & \% de acidez \\
\hline Picual & 0,98 \\
\hline Azeradj & 0,85 \\
\hline Sevillana & 0,71 \\
\hline Manzanilla & 0,76 \\
\hline Pendolino & 0,69 \\
\hline Campanil & 0,66 \\
\hline
\end{tabular}

\section{2.- Indice de peróxidos}

Los valores de peróxidos mostrado en el cuadro se encuentran elevados, quizá se debe al método de extracción utilizada, que presentaban problemas de sobrexposición al medio ambiente, sin embargo todos los aceites se encuentran en función a la norma, aunque su durabilidad puede verse afectada.

Tabla $\mathrm{N}^{\circ}$ 04. Índice de Peróxidos de las muestras

\begin{tabular}{|l|r|}
\hline \multicolumn{1}{|c|}{ Variedad } & $\%$ \\
\hline Sevillana & 13,8 \\
\hline Azaradaj & 12,3 \\
\hline Picual & 17,9 \\
\hline Campanil & 7,2 \\
\hline Pendolino & 11,4 \\
\hline Manzanilla & 9,3 \\
\hline
\end{tabular}

\section{3.- Absorbancia en el ultravioleta}

Los valores de las muestras de las diferentes variedades ofrecidas en la Tabla $\mathrm{N}^{\circ} 07$ se encuentran dentro de la norma en lo que se refiere al $\Delta K$ y $K_{270}$, pues indica su pureza. Cabe señalar el mejor valor es el pendolino.

Tabla $\mathbf{N}^{\circ} \mathbf{0 5}$. Absorbancia Ultravioleta

\begin{tabular}{|l|c|r|c|c|r|}
\hline Variedad & $\mathbf{2 3 2}$ & \multicolumn{1}{c|}{$\mathbf{2 6 6}$} & \multicolumn{1}{c|}{$\mathbf{2 7 0}$} & $\mathbf{2 7 4}$ & \multicolumn{1}{c|}{$\mathbf{\Delta K}$} \\
\hline Manzanilla & 0,430 & 0,0258 & 0,025 & 0,024 & 0,00011 \\
\hline Picual & 1,113 & 0,128 & 0,122 & 0,035 & 0,00014 \\
\hline Pendolino & 0,795 & 0,0375 & 0,0353 & 0,033 & $-2,057 \mathrm{E}-05$ \\
\hline Sevillana & 1,349 & 0,187 & 0,187 & 0,180 & 0,0039 \\
\hline Azerdaj & 1,272 & 0,147 & 0,147 & 0,141 & 0,00297 \\
\hline Campanil & 1,292 & 0,143 & 0,143 & 0,130 & 0,0021 \\
\hline
\end{tabular}

\section{C.-ANÁLISIS SENSORIAL (CATA DE ACEITE) DE LAS VARIEDADES ESTUDIADAS.}

\section{1.-Picual}

Del formato de evaluación sensorial empleado para la variedad Picual (Anexos, Gráfico $\mathrm{N}^{\circ} \mathrm{O} 1$ ), podemos indicar que esta variedad presentó un sabor ligero a aceitunas verde, acompañado de un atributo de sabor, muy intenso, muy picante, ligeramente dulce, marcada astringencia y un almendrado suave, ningún defecto. Por lo tanto, con una calificación global de 7 puntos.

\section{2.-Campanil:}

Presenta un frutado aceituna verde suave $y$ más intensamente a otras frutas maduras, muy 
picante, ligeramente dulce, astringencia muy perceptible rematantado con una suave atributo a almendras.

\section{3.-Pendolino}

Ofrece un ligero sabor a manzana y fuerte sabor a ciruela, un suave picante y ligeramente dulce, con la presencia de un ligero sabor a frambuesas. Cero defectos. En la globalización presenta un valor de 7 .

\section{4.-Sevillana Injerto}

Tiene un mediano sabor a aceitunas verdes, ligeramente menor a otras frutas y perceptiblemente picante, con una suave astringencia y sabor medio a almendras.

\section{5.-Azeradj}

Ofrece un sabor medio a frutas, una astringencia casi imperceptible, con una presencia media de sabor a madera. Cero defectos y una evaluación global de 7 puntos. Cero defectos. Color anormal pardo anaranjado.

\section{6.- Manzanilla}

Contiene un sabor muy marcado a almendras y una combinación muy suave de atributos a hojas, amargo y astringente; mezclado con atributo amargo y dulce, ligeramente más intenso que el anterior. Cero defectos. Puntaje en la evaluación global de 7.

\section{V.- CONCLUSIONES}

- Las variedades estudiadas presentan una baja cantidad de aceite, excepto la variedad Sevillana y Pendolino.

- Todas las variedades de aceitunas estudiadas presentan características de aceite de oliva extra virgen, en cuanto a su acidez y el análisis sensorial.

- En cuanto al índice de Peróxidos, en algunas muestras es elevado, pero están dentro de los límites de la norma.

- El color de los aceites obtenidos son aceptables excepto el proveniente del Azaradj que tuvo una coloración pardo naranja.

- Las variedades presentan un comportamiento diferencial en cuanto a calidad del aceite comparado a su origen.

\section{VI.-RECOMENDACIONES}

- Realizar estudios sobre otras variedades para determinar sus potencialidades.

- Realizar estudios sobre la vida útil de lo aceites de las variedades de aceites estudiadas

\section{REFERENCIAS BIBLIOGRÁFICAS}

- Alférez, R; Robles, M. (2007). Caracterización morfológica de trece variedades de Olivo (Olea europeae $L$ ) introducidas en el germoplasma del INPREX-Tacna. COIN-UNJBG.

- Aranda, Z R. (2001) .Ingeniería en decantadores para un estudio de factibilidad de una planta procesadora de aceite de oliva en la irrigación de la Yarada .UNJBG-FAIA. Tesis para optar el Titulo Profesional de ingeniera en industrias Alimentarias, UNJBG_Tacna.

- Boskou, Dimitrios (1998).Química y Tecnología de aceite de Oliva. Editorial Mundi prensa.Madrid. España

- Díaz, A (1988) Diagnostico de la producción de aceitunas de mesa en el Perú.

- De Florio, E. (2009). Visita de Campo al Banco de Germoplasma del IMPREX. Informe no publicado.

- Earlie R, L. (1998) Ingeniería de los Alimentos. Editorial Acriba. Zaragoza.

- Enciclopedia Libre Universal. Olivo. Bajado el día 24 de Abril del 2009 de la pagina.

- Conservas Kilimanjaro (2009) Revista virtual. Disponible en www. conservaskilimanjaro.com /aceitunas.php

- Lanchipa Bergamini Liliana (2003) Evaluación de la Calidad Químico -Sensorial de los aceites de Oliva virgen mono varietales d e Tacna. Tesis Optar grado de Magíster en Ciencia y Tecnología de Alimentos .ESPG-UNJBG. Tacna. Perú.

- Leo, R, E. (2000) Diagnostico de la Calidad del Aceite de Oliva Tacneño Virgen según el Proceso Productivo. Tesis Optar Titulo de Ingeniero. FAIA-UNJBG.

- Loussert, R; Brousse, G. 1980 .El Olivo. 
Madrid.

- Frías, L; et al ( 1991) Analista de Laboratorio de Alzamara. Conserjería de Agricultura y Pesca de andalucía. .Colección Apuntes n ${ }^{\circ}$ 6/1991. Sevilla.

-FAO.WWW.Fao.org Página Web de la FAO

- IMPREX. (2009) Entrevista personal con el jefe de campo.

- Ministerio de Agricultura Boletín Mensual 2009.

\section{REFERENCIAS ELECTRÓNICAS}

- Internacional-olive-oil :

http://www.internationaloliveoil.org/resgen/ esp/Espa\%C3\%B1a / pomologica.pdf

\section{Correspondencia:}

Enrique A. De Florio Ramirez

ede_florio@hotmail.com

Ciudad Universitaria fundo "Los Granados"

Av. Miraflores s/n Tacna - Perú

Liliana Lanchipa Bergamini inei100000@hotmail.com Ciudad Universitaria fundo "Los Granados" Av. Miraflores s/n Tacna - Perú

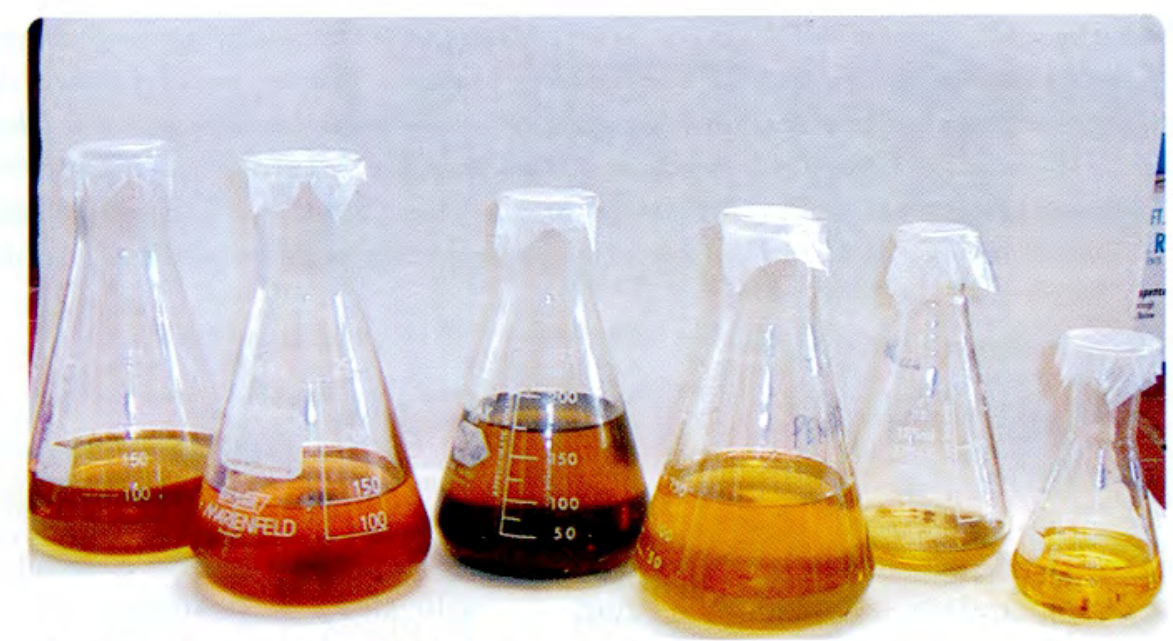

Muestras de Aceites de variedades ensayadas 